\title{
Nanostructured diatom frustule immunosensors
}

\author{
Gregory L Rorrer*1 and Alan X Wang ${ }^{2}$ \\ ${ }^{1}$ School of Chemical, Biological, and Environmental Engineering, Oregon State University, Corvallis, Oregon 97331, USA \\ ${ }^{2}$ School of Electrical Engineering and Computer Science, Oregon State University, Corvallis, Oregon 97331, USA
}

\section{Commentary}

A major challenge for immunocomplex based biosensors is labelfree detection [1] as Enzyme-Linked Immunosorbent Assay (ELISA) or other chemical labeling techniques are typically required for optical signal generation. Current label-free platforms for immuno-complex based sensing rely on Surface Plasmon Resonance (SPR), piezoelectric, electrochemical, or impedance measurements, all of which require specialized instrument packages. Furthermore, many label-free detection methods suffer from low sensitivity. Recently, our research has made exciting new advances in the development of nanostructured biogenic materials to serve as a platform for sensitive and selective detection of immunocomplex formation based on stand-off, label-free optical reporting. The transducer element relies on the hierarchical structure of diatom biosilica, its ease of functionalization, and its optical properties.

Diatoms are single-celled photosynthetic algae that fabricate microscopic shells called frustules. The frustules are composed of nanostructured, biogenic silica ordered into intricately patterned, defined pore arrays, and fabricated through a cell-mediated, biomineralization process. A unique feature of diatom metabolism is that dissolved silicon is a required substrate for cell wall biosynthesis and subsequent cell division. Unlike mesoporous silicates, diatom frustules can assume a microscale, disk-like form factor with a flat surface possessing a periodic pore structure hierarchically ordered at the submicron and nanoscales (Figure 1). The pore array can act as a photonic crystal to enhance wavelength-specific guided modes of light absorption. Furthermore, the ambient silica biomineralization

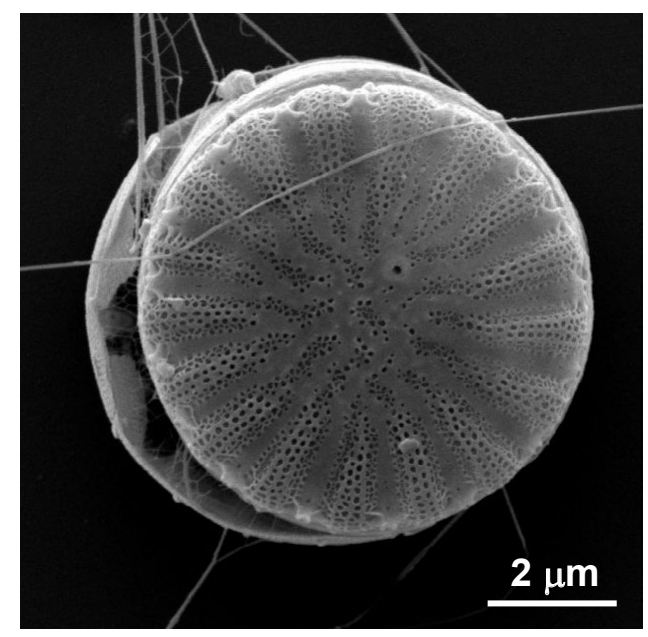

Figure 1. Scanning electron microscopy (SEM) image of whole Cyclotella diatom, showing frustule pore detail. process imparts defects in the biogenic silicon oxide structure, such as non-bridging oxygen centers (Si-O:) and silanol (Si-OH) (silanol) groups. These defects lower the conduction band of the material to enable spontaneous emission of blue light (400-450 nm) under excitation by ultraviolet light of a higher energy, a process known as photoluminescence. Through controlled cell cultivation, the level of detail in the diatom frustule nanostructure can be increased, which in turn increases the defect density and the intensity of the blue photoluminescence emission [2].

The surface silanol groups on the diatom biosilica pore array can be readily functionalized with biomolecules such as antibodies that serve as recognition elements for biosensing [3]. A typical functionalization scheme is shown in Figure 2. In this scheme, silanol groups are derivatized to amine groups with 3-aminopropyltrimethoxysilane (APTMS). The antibody is then covalently attached through a bifunctional crosslinking reagent which couples the surface amine group to an amine group on one of amino acid residues (e.g. lysine) on the antibody. Diatom frustule nanostructures may be particularly attractive for bionanotechnology applications, given the current interest in new approaches for biomolecule nanopatterning [4] with an eye towards microscale "lab-on-chip" applications.

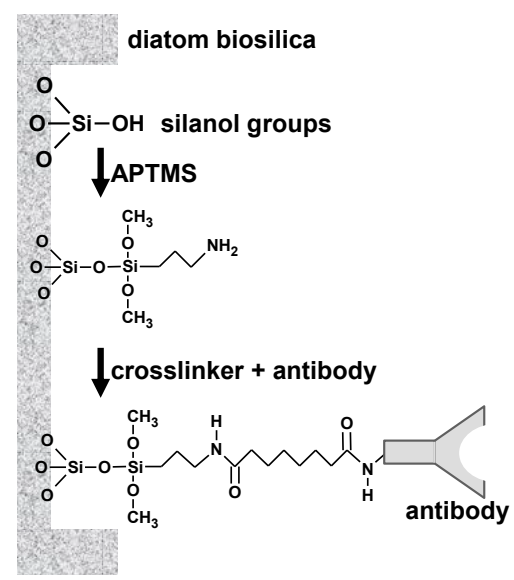

Figure 2. Covalent bonding of antibody to silanol groups on diatom biosilica.

Correspondence to: Gregory L. Rorrer, School of Chemical, Biological, and Environmental Engineering, Oregon State University, Corvallis, Oregon, 97331, USA; E-mail: Gregory.Rorrer@oregonstate.edu

Received: May 02, 2016; Accepted: May 10, 2016; Published: May 13, 2016 
The photoluminescent properties nanoscale semiconductor materials change upon their interaction with biomolecules [5]. This phenomenon may serve as the basis for the label-free optical detection of immunocomplex formation. Towards this end, we have shown that covalent attachment of antibodies to the diatom frustule surface increases the photoluminescence emission from the diatom biosilica $[3,6]$. This is due to the nucleophilic amino acid residues on the antibody, which donate electrons to non-radiative defect sites on the diatom biosilica surface, thereby decreasing nonradiative electron decay and increasing radiative emission. The binding of a nucleophilic antigen with its complimentary antibody further enhances this photoluminescence emission, enabling selective detection of immunocomplex formation. In contrast, the binding of an electrophilic antigen with its complimentary antibody quenches the photoluminescence emission, which also enables selective detection. In general, the sensitivity of the response is based on the proportion of nucleophilic or electrophilic sites on the antigen relative to the antibody, as schematically illustrated in Figure 3.

Two examples from our research with antibody-functionalized diatom frustules demonstrate photoluminescence enhancement upon binding with a nucleophilic antigen, and photoluminescence quenching upon binding with an electrophilic antigen (Figure 3 ). The first example illustrates the selective detection of a large nucleophilic biomolecule through enhanced photoluminescence [3]. Frustule biosilica was isolated from cultured cells of the centric diatom Cyclotella, which has a diameter of nominally $10 \mu \mathrm{m}$. The model antibody rabbit immunoglobulin $\mathrm{G}(\mathrm{IgG})$ was covalently attached to the frustule biosilica by silanol amination and crosslinking steps to a surface site density of approximately $4000 \mathrm{IgG}$ molecules per $\mu \mathrm{m}$ of valve surface area. Functionalization of the diatom biosilica with the nucleophilic IgG antibody amplified the intrinsic blue photoluminescence of diatom biosilica by a factor of six. Furthermore, immunocomplex formation with the complimentary antigen anti-rabbit IgG further increased the peak photoluminescence intensity by at least a factor of three, whereas a non-complimentary antigen (goat anti-human IgG) did not. The enhancement in blue photoluminescence emission was correlated to the antigen concentration, where immunocomplex binding was described by a Langmuir isotherm with a binding constant of $2.8 \times 10^{-7}$ $\mathrm{M}$, which was comparable to known binding constant.

The second example illustrates selective detection of a small electrophilic molecule through partial quenching of photoluminescence [6]. Explosive compounds are particularly challenging to detect [7]. The target molecule of interest in the explosive 2,4,6 trinitrotoluene (TNT, molecular weight $227 \mathrm{~g} / \mathrm{mole}$ ). TNT is an electrophilic molecule due to its three electron-deficient nitro $\left(-\mathrm{NO}_{2}\right)$ groups. Frustule biosilica was isolated from cultured cells of the pennate diatom Pinnularia sp. $(5 \mu \mathrm{m}$ width by $30 \mu \mathrm{m}$ length) and functionalized with a single chain variable fragment $(\mathrm{scFv})$ derived from an anti-TNT monoclonal antibody via silanol amination and crosslinking steps as described earlier. When TNT was bound to the anti-TNT scFv-functionalized diatom frustule biosilica, the photoluminescence emission from the biosilica was partially quenched (about 15\%) due to the electrophilic nature of the nitro $\left(-\mathrm{NO}_{2}\right)$ groups on the TNT molecule. The dose-response curve for immunocomplex formation of TNT on the scFv-functionalized diatom frustule biosilica was described by a Langmuir isotherm and had a halfsaturation binding constant of $6.4 \times 10^{-8} \mathrm{M}$ and statistically-significant measured detection limit of $3.5 \times 10^{-8} \mathrm{M}$. The binding was selective for TNT and TNB (trinitrobenzene) but not other munitions constituents, including RDX (hexahydro-1,3,5-trinitro-1,3,5-triazine) or 2,6-DNT (2,6-dinitrotoluene).

We have also shown that diatom frustules decorated with silver nanoparticles enhance the sensitivity of surface-enhanced Raman scattering (SERS) for detection of immunocomplex formation [8]. Frustule biosilica isolated from cultured cells of the pennate diatom Pinnularia sp. was surface functionalized with amine groups as described above. The amine groups served as nucleation sites for self-assembly of silver nanoparticles onto the frustule surface. The antibody (goat antimouse IgG) was then attached directly onto the silver nanoparticles through the thiol groups as well as through ionic, hydrophilic and hydrophobic interactions. To demonstrate the potential of diatom frustule enhanced SERS, this antibody-functionalized diatom frustule was challenged with gold nanoparticle labeled mouse IgG. Although this was not a label-free detection mode, the diatom frustules improved the detection limit of mouse IgG to $100 \mathrm{pg} / \mathrm{mL}\left(6.25 \times 10^{-13} \mathrm{M}\right)$, which is about $100 \mathrm{x}$ better than the same immumocomplex immobilized onto a nonporous glass surface. The enhancement was attributed to the periodic sub-micron scale pore structures of diatom frustule, which enabled guided-mode resonance at visible wavelengths to enhance the SERS signals, similar to artificial photonic crystals (Figure 4).

In summary, diatom frustules offer a versatile platform for sensitive and selective immunocomplex biosensing. The key features of this platform are the intrinsic blue photoluminescence emission of the nanostructured biosilica combined with ease of antibody functionalization of this material. Interaction of target molecules with antibody-functionalized diatom biosilica allows for selective detection of immunocomplex formation through enhancement of the photoluminescence emission by nucleophilic antigens or quenching of the photoluminescence emission by electrophilic antigens.
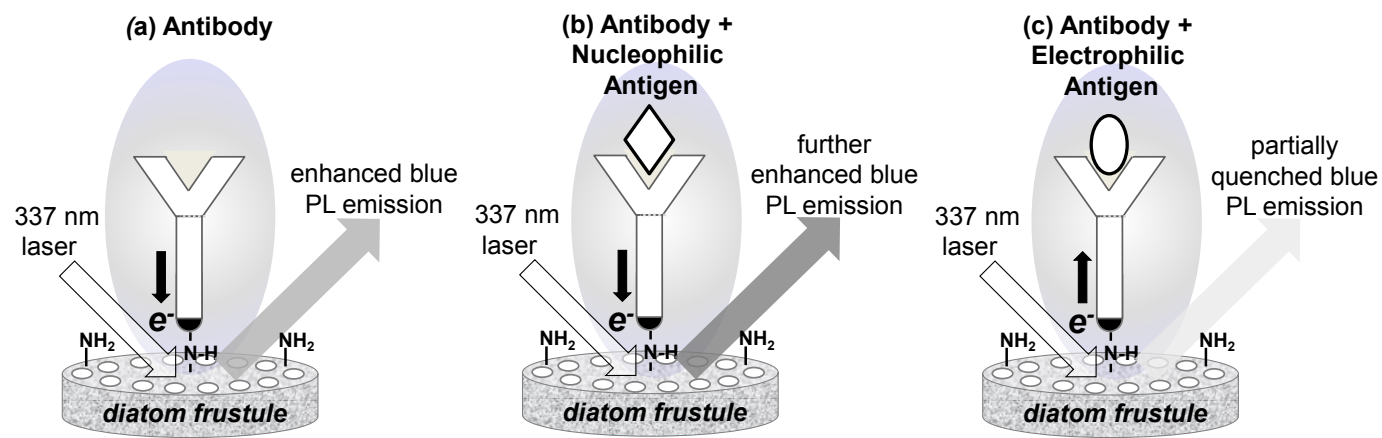

Figure 3. Potoluminescence emission of diatom biosilica upon (a) antibody functionalization, (b) immunocomplex formation with a nucleophilic antigen, and (c) immunocomplex formation with an electrophilic antigen. 

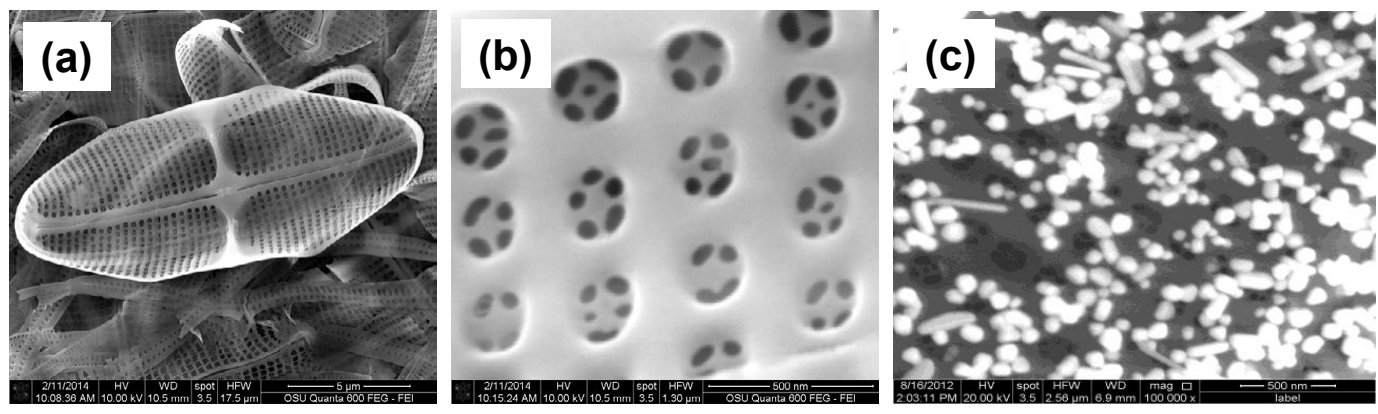

Figure 4. SEM images of Pinnularia diatom, showing (a) overall frustule, (b) hierarchical pore detail, and (c) silver nanoparticles self-assembled on surface.

\section{References}

1. Holford TR, Davis F, Higson SP (2012) Recent trends in antibody based sensors Biosens Bioelectron 34: 12-24. [Crossref]

2. Qin T, Gutu T, Jiao J, Chang CH, Rorrer GL (2008) Photoluminescence of silica nanostructures from bioreactor culture of marine diatom nitzschia frustulum $J$ Nanoscience Nanotechnol 8: 2392-2398.

3. Gale DK, Gutu T, Jiao J, Chang CH, Rorrer GL (2009) Photoluminescence detection of biomolecules by antibody-functionalized diatom biosilica Adv Funct Mat 19: 926-933.

4. Mendes PM, Yeung CL, Preece JA (2007) Bio-nanopatterning of Surfaces Nanoscale Res Lett 2: 373-384. [Crossref]
5. Sapsford KE, Pons T, Medintz IL, Mattoussi H (2006) Biosensing with luminescent semiconductor quantum dots Sensors 6: 925-953

6. Zhen L, Ford N, Gale DK, Roesijadi G, Rorrer GL (2016) Photoluminescence detection of 2,4,6-trinitrotoluene (TNT) binding on diatom frustule biosilica functionalized with an anti-TNT monoclonal antibody fragment Biosens. Bioelectron 79: 742-748.

7. Smith RG, D'Souza N, Nicklin S (2008) A review of biosensors and biologicallyinspired systems for explosives detection Analyst 133: 571-584. [Crossref]

8. Yang J, Zhen L, Ren F, Campbell J, Rorrer GL, et al. (2015) Ultra-sensitive immunoassay biosensors using hybrid plasmonic-biosilica nanostructured materials $J$ Biophotonics 8: 611-687.

Copyright: (C2016 Rorrer GL. This is an open-access article distributed under the terms of the Creative Commons Attribution License, which permits unrestricted use, distribution, and reproduction in any medium, provided the original author and source are credited. 